\title{
Bone morphogenetic protein-7 expression reflects the high proliferative ability and aggressiveness of thymic epithelial tumors
}

\author{
Kentaro Miura ${ }^{1}$, Kazutoshi Hamanaka ${ }^{1}$, Tsuyoshi Uehara ${ }^{2}$, Masanobu Momose ${ }^{2}$, Yukiko Kanai ${ }^{1}$, \\ Shunichiro Matsuoka ${ }^{1}$, Tetsu Takeda ${ }^{1}$, Hiroyuki Agatsuma ${ }^{1}$, Akira Hyogotani ${ }^{1}$, Ken-ichi Ito ${ }^{3}$ \\ ${ }^{1}$ Division of General Thoracic Surgery, Department of Surgery, ${ }^{2}$ Department of Laboratory Medicine, ${ }^{3}$ Division of Breast and Endocrine Surgery, \\ Department of Surgery, Shinshu University School of Medicine, 3-1-1 Asahi, Matsumoto, Japan \\ Contributions: (I) Conception and design: K Miura, K Ito, T Uehara; (II) Administrative Support: K Miura, K Ito; (III) Provision of study materials \\ or patients: K Miura, K Hamanaka, S Matsuoka, T Takeda, H Agatsuma, A Hyogotani; (IV) Collection and assembly of data: K Miura, M Momose, \\ Y Kanai; (V) Data analysis and interpretation: K Miura, T Uehara; (VI) Manuscript writing: All authors; (VII) Final approval of manuscript: All \\ authors. \\ Correspondence to: Ken-ichi Ito. Division of Breast and Endocrine Surgery, Department of Surgery, Shinshu University School of Medicine, 3-1-1 \\ Asahi, Matsumoto, Nagano, 390-861, Japan. Email: kenito@shinshu-u.ac.jp.
}

Background: Bone morphogenetic protein-7 (BMP-7) is a transforming growth factor- $\beta$ superfamily member. We examined whether BMP-7 expression in thymic epithelial tumors is associated with their clinicopathological features.

Methods: One hundred and thirty-two clinical specimens were analyzed in this study. The expression of BMP-7 was detected using immunohistochemistry and was scored as $0,1,2$, or 3 according to its intensity and was then classified as negative (score 0 and 1) or positive (2 and 3). In addition, Ki-67 staining was performed in type B3 thymoma and thymic cancer.

Results: The positive ratio of BMP-7 was $80 \%$ in thymic cancer and $70 \%$ in thymoma type B3. In contrast, the positive ratios of BMP-7 in type B2 (29.1\%), B1 (3.7\%), AB (26\%), and A (31\%) were relatively low. The mean $\mathrm{Ki}-67$ labeling index of the BMP-7 positive group $(10.1 \% \pm 5.9 \%)$ was significantly higher than that of the BMP-7 negative group $(4.9 \% \pm 5.9 \%)$ in type B3 thymoma and thymic cancer $(\mathrm{P}=0.012)$. The BMP7 positive group showed significantly poorer overall survival (OS) than the BMP-7 negative group across all patients with thymic epithelial tumors and in all types of thymomas $(\mathrm{P}=0.006, \mathrm{P}=0.018)$; however, no difference was observed in thymic cancers.

Conclusions: This study showed that high expression of BMP-7 correlated with a poor prognosis in patients with thymic epithelial tumors, and the expression of BMP-7 was higher in type B3 thymomas and thymic cancers than in other types of thymomas. BMP-7 might serve as a novel prognostic biomarker for thymic epithelial tumors.

Keywords: Bone morphogenetic protein-7 (BMP-7); thymic epithelial tumors; thymoma; thymic cancer

Submitted Sep 12, 2019. Accepted for publication Dec 16, 2019.

doi: $10.21037 /$ jtd.2020.01.14

View this article at: http://dx.doi.org/10.21037/jtd.2020.01.14

\section{Introduction}

Thymic epithelial tumors are among the most common neoplasms in the anterior mediastinum. At least 24 histomorphological classifications and 14 staging systems have been proposed in the past century (1). The World
Health Organization (WHO) classification and Masaoka staging, which reflect the histological type, clinical findings, and prognosis, are used most commonly worldwide for the classification of thymic epithelial tumors at present (2). However, as thymic epithelial tumors show a variety of morphologies and there are many unclear points regarding 
their biological behaviors, the classification of thymic epithelial tumors remains challenging. Although several factors, such as podoplanin, p53, Ki-67, and GLUT-1, have been reported to be associated with the individual biological behavior of thymic epithelial tumors (3-7), more unique markers which reflect the biology of thymic epithelial tumors are required. In order to detect the expression of molecules that are important for the progression of thymic epithelial tumors, we compared gene expression profiles using microarray analysis between the normal thymus and type B2 thymoma in clinical specimens obtained in our department. We found that the expression of $B M P-7$ in thymoma type B2 increased 11.8 folds higher than that in the normal thymus.

Bone morphogenetic protein-7 (BMP-7) belongs to the transforming growth factor $\beta$ (TGF- $\beta$ ) superfamily. BMP-7 was first identified as a human homolog of the bovine osteogenic proteins, and designated as osteogenic protein-1 (OP-1) (8). Experiments using knockout mouse models of the $B M P-7$ gene have shown that BMP-7 is indispensable in kidney development, as well as in the morphogenesis of fingers in the hind limbs, ribs, craniofacial bones, and eyes (9-11). Subsequent studies have demonstrated that BMP7 is expressed in various tissues, such as the kidney, brain, and skeletal muscle, and is involved in apoptosis, migration, and cell proliferation during human development $(12,13)$. In addition, several reports have demonstrated relationships between the overexpression of BMP-7 and the prognosis of the patients with various malignancies, such as gastric cancer, colorectal cancer, hepatocellular carcinoma, and melanoma (14-17). However, the association between BMP-7 expression and the clinicopathological features of thymic epithelial tumors has not been elucidated.

The aims of this study were to elucidate whether BMP-7 expression is related to the clinicopathological features of thymic epithelial tumors, and to explore the utility of BMP-7 as a novel biomarker of thymic epithelial tumors.

\section{Methods}

\section{Patient population and study design}

The specimens were obtained from 182 patients with thymic epithelial tumors who underwent surgery at Shinshu University Hospital from 1998 to 2015. Fifty specimens from patients who received preoperative chemotherapy, radiation therapy, or who underwent incomplete resection were excluded, and 132 specimens were analyzed in this study.

Using a database, we analyzed the association between the expression of BMP and clinicopathological characteristics of the patients, including age, sex, tumor size, the WHO classification, Masaoka stage, primary tumor standard uptake value max (SUV-max) on fluorodeoxyglucose positron emission tomography (FDGPET), and the presence or absence of comorbid myasthenia gravis. This study was approved by our Institutional Research Ethics Committee (No. 542).

\section{Immunobistochemical analyses}

The specimens of epithelial thymic tumor were paraffinembedded and cut into $4 \mu \mathrm{m}$ sections. They were deparaffinized using xylene and ethanol. The endogenous peroxidase activity was blocked using methanol and 30\% $\mathrm{H}_{2} \mathrm{O}_{2}$ solution for $30 \mathrm{~min}$ at room temperature. After protein blocking using $1 \%$ PSA/PBS for $1 \mathrm{~h}$ at room temperature, specimens were incubated overnight at 44 with a primary antibody against human BMP-7 (1:100, R\&D Systems Inc., Minneapolis, MN, USA) and human Ki-67 (MIB-1, 1:100, DAKO, Glostrup, Denmark). The sections were then washed in PBS 3 times, and probed with an anti-mouse IgG antibody labelled with Histofine Simple Stain MAX-PO (Nichirei, Tokyo, Japan) for $1 \mathrm{~h}$ at room temperature. The sections were washed 3 times in PBS for 5 min each time and the immune complex was visualized using Histofine Simple Stain DAB (Nichirei). After washing in water, the sections were counterstained with hematoxylin and then mounted.

The immunoreactivity was scored and reviewed by 1 pathologist (UT) and 1 thoracic surgeon $(\mathrm{KM})$ who were blinded to the clinical characteristics. A normal renal tubule was used as a positive control for BMP-7 staining. Because almost all specimens were stained homogenously, BMP-7 staining was classified into 4 groups based on intensity alone; 0, 1 (weak), 2 (moderate), and 3 (strong; equal to positive control). Scores of 0 and 1 were considered negative, and 2 and 3 were positive. Ki-67 expression was evaluated based on the percentage of cells showing nuclear staining among 500 tumor cells (magnification $\times 400$ ).

\section{Statistical analysis}

The categorical variables were analyzed using Chi-square tests, and continuous variables were analyzed using a Student's $t$-test. The results are presented as mean \pm 
Table 1 Patients' characteristics

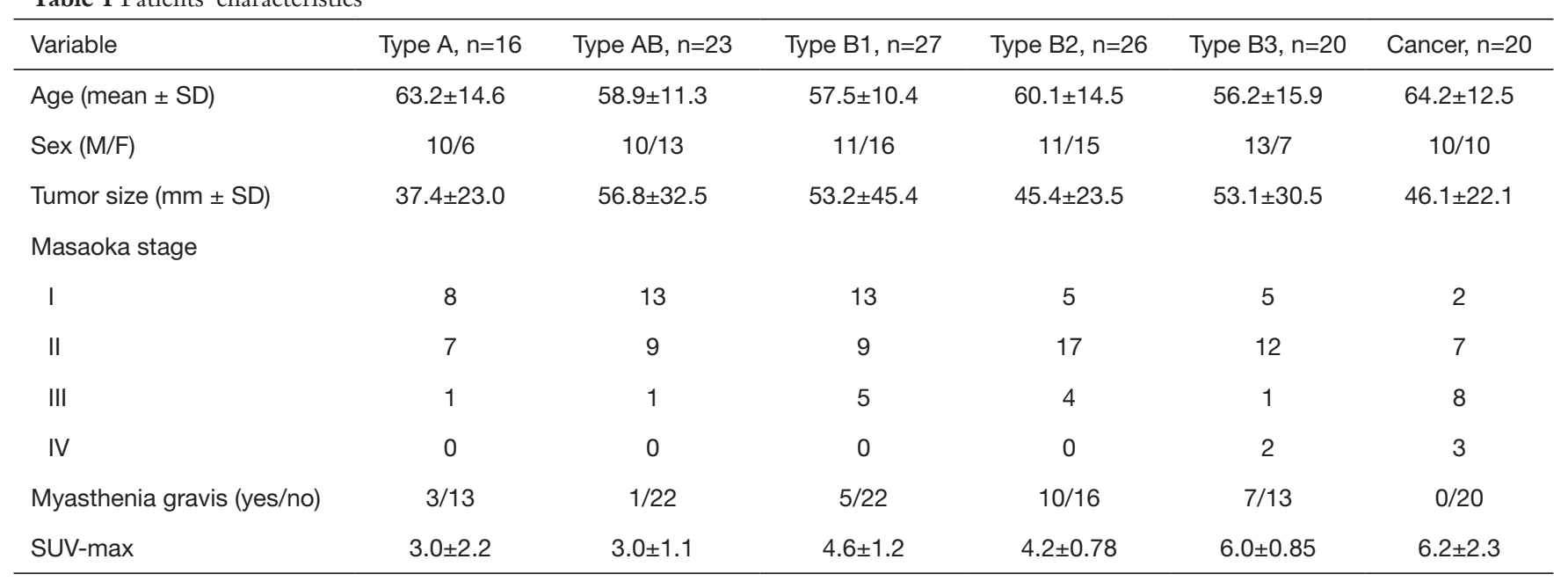

SD, standard deviation; SUV-max, standard uptake value max.

standard deviation. Overall survival (OS) was evaluated using the Kaplan-Meier method, and the differences between each group were calculated using the log-rank test. Multivariate analysis was performed using Cox proportional hazard regression models. All statistical analyses were performed using SPSS (version 32, Chicago, IL, USA). A P value $<0.05$ was considered statistically significant.

\section{Results}

\section{Patient selection and characteristics}

One hundred and thirty-two patients were classified according to the WHO classification as follows: type A $(n=16)$, type $A B(n=23)$, type B1 $(n=27)$, type B2 $(n=26)$, type $B 3(n=20)$, and thymic cancer $(n=20)$. Table 1 shows the clinical characteristics of thymic epithelial tumors according to the WHO classification. Masaoka stage IV tumors were observed in the type B 3 and thymic cancer group, and comorbid myasthenia gravis was not observed in the thymic cancer group.

\section{Immunobistochemical staining of BMP-7}

Figure 1 shows the representative immunohistochemical staining of BMP-7 in the thymic epithelial tumors. Figure 2 shows the expression of BMP-7 in thymic tumors according to the $\mathrm{WHO}$ classification. The positive ratio of BMP-7 was $80 \%(16 / 20)$ in thymic cancer and $70 \%(14 / 20)$ in thymoma type B3. In contrast, the positive ratios of BMP-7 in type B2, $\mathrm{B} 1, \mathrm{AB}$, and A were relatively low (B2: 29.1\%, B1: 3.7\%,
$\mathrm{AB}: 26 \%$, and $\mathrm{A}: 31 \%)$. In particular, type B1 showed a lower positive ratio of BMP-7 than other types (Figure 2).

Table 2 shows the relationships between BMP-7 expression and the clinicopathological characteristics of 132 patients with thymic epithelial tumors. The number of patients with BMP-7 positive tumors was 49 and that of patients with BMP-7 negative tumors was 83 . The ratios of BMP-7 positive tumors were higher in type $\mathrm{B} 3$ and thymic cancer than in other types of thymoma, and the ratios of BMP-7 positive tumors were significantly associated with the degree of malignancy stratified according to the WHO classification $(\mathrm{P}<0.001)$. The SUV-max on FDG-PET was significantly higher in the BMP-7 positive group than in the BMP-7 negative group $(\mathrm{P}=0.038)$. In contrast, there was no significant difference between the 2 groups with regard to tumor size and Masaoka stage $(\mathrm{P}=0.897, \mathrm{P}=0.374)$ (Table 2).

\section{Immunobistochemical staining of Ki-67}

As Ki-67 has been demonstrated to be associated with the aggressiveness of thymic epithelial tumors, we performed immunohistochemical analysis of $\mathrm{Ki}-67$ to evaluate the association between the BMP-7 expression in thymoma type B3 $(n=20)$ and thymic cancer $(n=20)$. We did not evaluate $\mathrm{Ki}-67$ staining in type $\mathrm{A}, \mathrm{AB}, \mathrm{B} 1$, and $\mathrm{B} 2$ thymomas because there were many Ki-67 positive cells among the infiltrated lymphocytes in these subsets of thymoma, which made the identification of Ki-67 positive tumor cells difficult. The mean Ki-67 labeling index of type B3 thymoma was $4.9 \% \pm 5.9 \%$, and that of thymic cancer was 

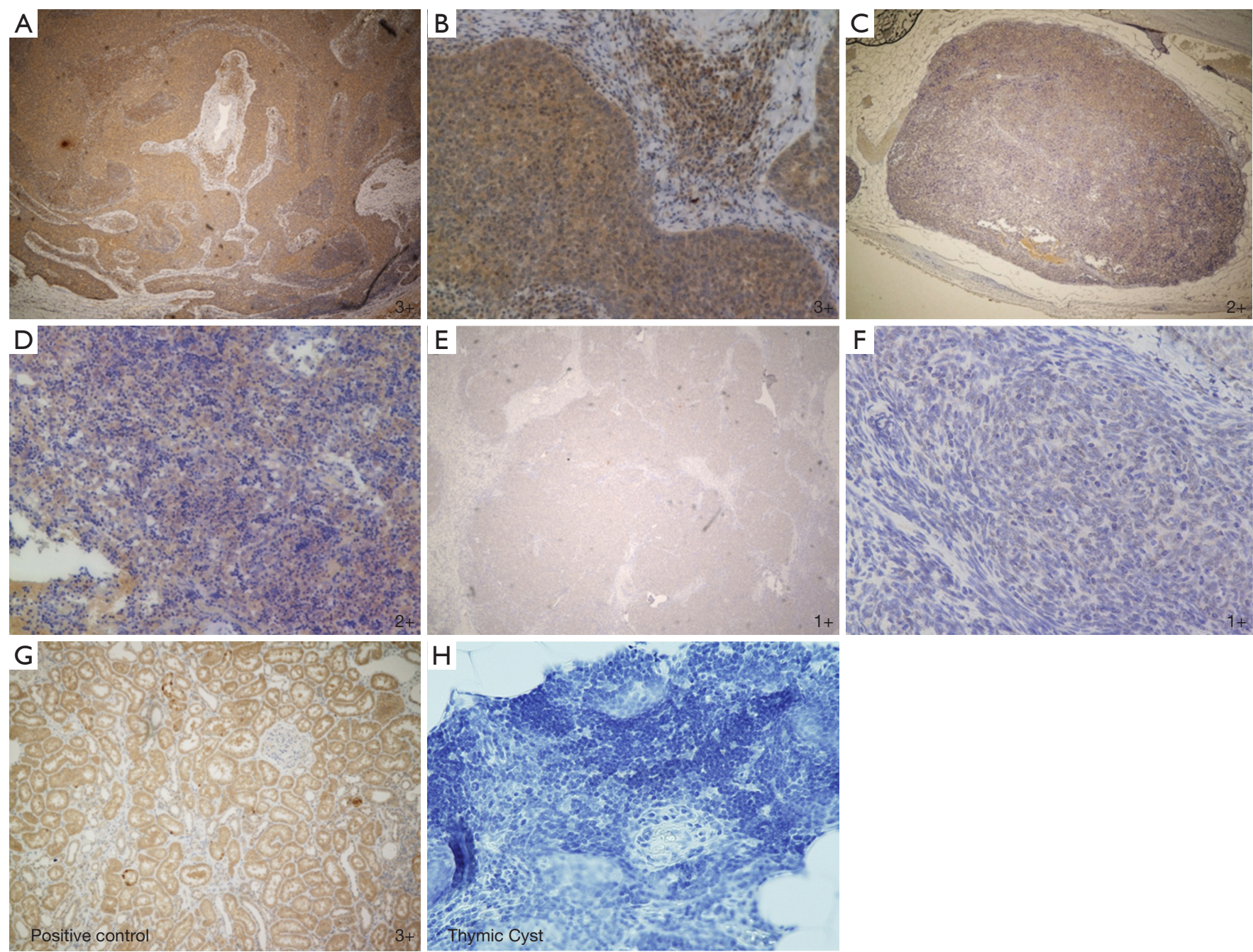

Figure 1 Representative findings of immunostaining of bone morphogenetic protein 7 (BMP-7). Immunostaining of BMP-7. (A) Thymic cancer, 3+, ×40; (B) thymic cancer, 3+, ×400; (C) thymoma B3, 2+, ×400; (D) thymoma B3, 2+, ×40; (E) thymoma A, 1+, ×400; (F) thymoma A, 1+, ×40; (G) human kidney tissue (positive control), 3+, ×400; (H) thymic cyst, $0, \times 400$.

$10.1 \% \pm 5.9 \%$ (Figure $3 A$ ). A significant difference between the 2 groups was observed $(\mathrm{P}=0.037)$. Furthermore, the mean Ki-67 labeling index of the BMP-7 positive group was $10.8 \% \pm 6.4 \%$, while that of the BMP-7 negative group was $6.5 \% \pm 5.4 \%$. Thus, the Ki-67 labeling index of the BMP7 positive group was significantly higher than that of the BMP-7 negative group ( $\mathrm{P}=0.026)$ (Figure 3B).

\section{Survival analysis}

Figure $4 A$ shows the OS of all patients with thymic epithelial tumors. The BMP-7 positive group demonstrated significantly poorer OS than the BMP-7 negative group
$(\mathrm{P}=0.006)$. Figure $4 B$ shows the Kaplan-Meier survival curve of all thymoma patients (type A-B3) and Figure $4 C$ shows Kaplan-Meier survival curve of thymic cancer patients. A significantly poorer prognosis was observed in BMP-7 positive patients than in BMP-7 negative patients in all thymoma patients; however, no difference in OS was observed in the patients with thymic cancer.

Univariate analyses showed that age, Masaoka stage, WHO classification, and BMP-7 expression were significant predictors of OS in all patients with thymic epithelial tumors. However, multivariate analyses using a Cox hazard model showed that age was the only independent prognostic factor $(\mathrm{P}=0.033)$ and $\mathrm{BMP}-7$ staining was not an 


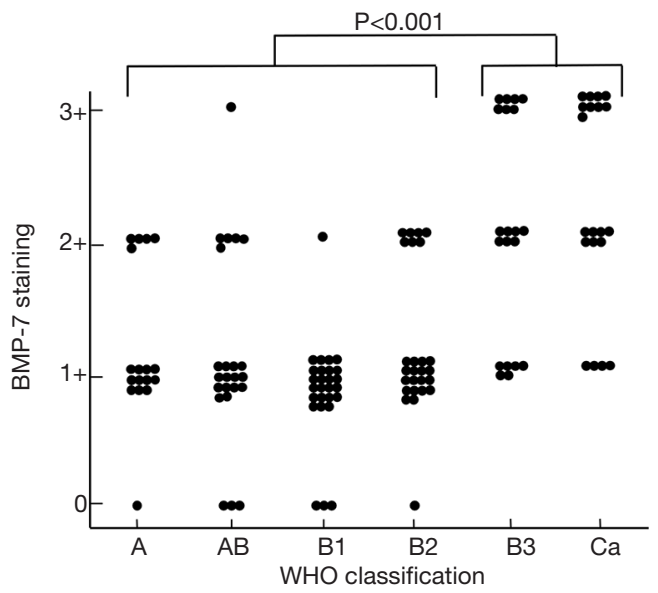

Figure 2 The expression of bone morphogenetic protein 7 (BMP-7) in each subgroup of thymic epithelial tumors. The positive rate of thymic cancer was $80 \%$, thymoma type B3: $70 \%$, B2: $29.1 \%$, B1: $3.7 \%$, AB: $26 \%$, A: $31 \%$. independent prognostic factor (Table 3).

\section{Discussion}

As thymic epithelial tumors have been classified according to their histopathologic features and staged based on the presence and extent of invasion, implants, lymph node involvement, or distant metastases, few biological markers are used in the differential diagnosis of thymic epithelial tumors. In the present study, we identified 2 novel findings regarding BMP-7 expression in thymic epithelial tumors. Firstly, BMP-7 expression was significantly higher in type $\mathrm{B} 3$ thymoma and thymic cancer than in type $\mathrm{A}, \mathrm{AB}, \mathrm{B} 1$, and B2 thymomas. Furthermore, patients with BMP-7 positive tumors showed a poorer prognosis than those with BMP-7 negative tumors. Secondly, BMP-7 expression was positively correlated with $\mathrm{Ki}-67$ staining in type B3

Table 2 Expression of bone morphogenetic protein 7 (BMP-7) and clinicopathological characteristics of thymic epithelial tumors

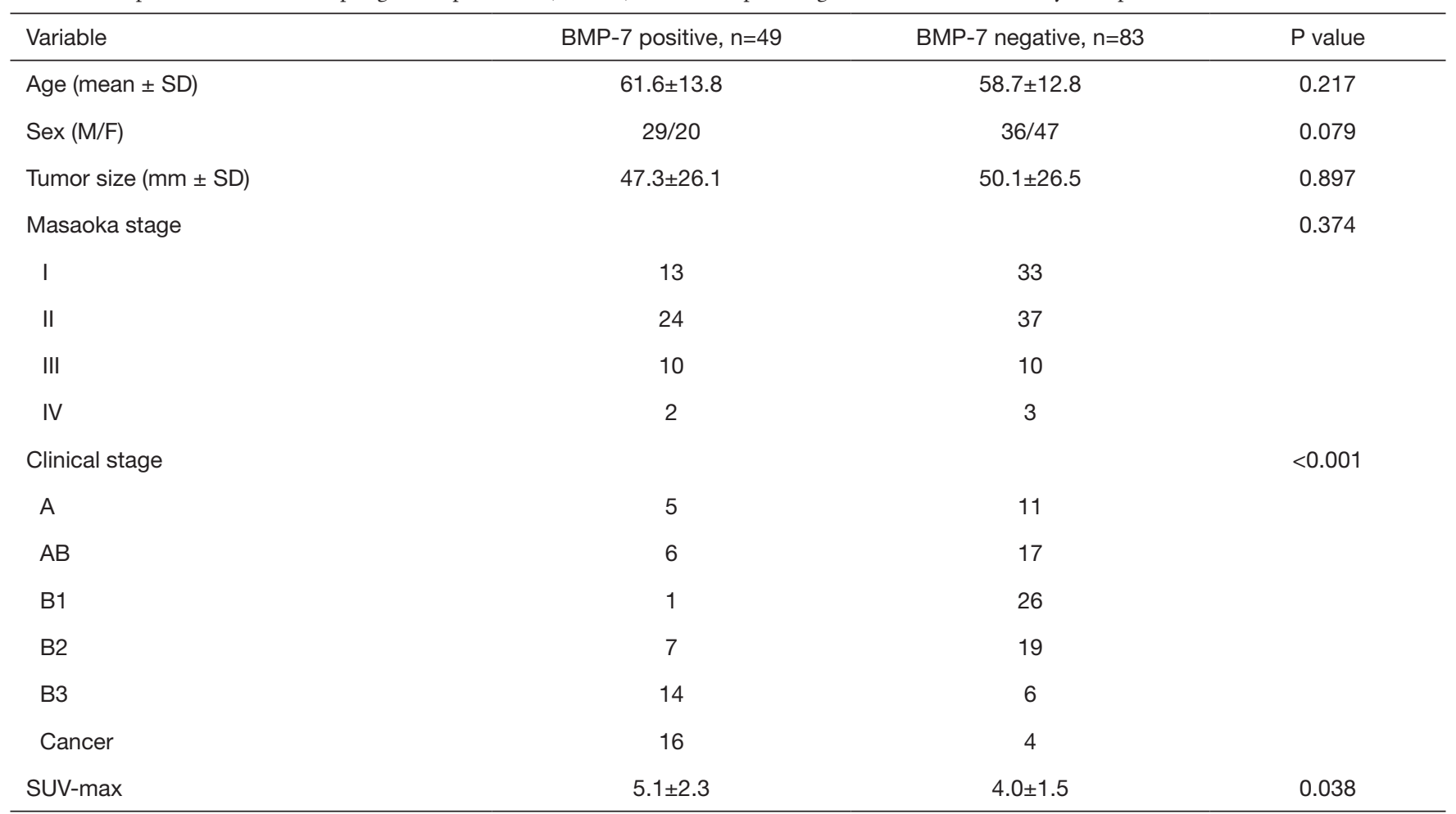

SD, standard deviation; SUV-max, standard uptake value max. 

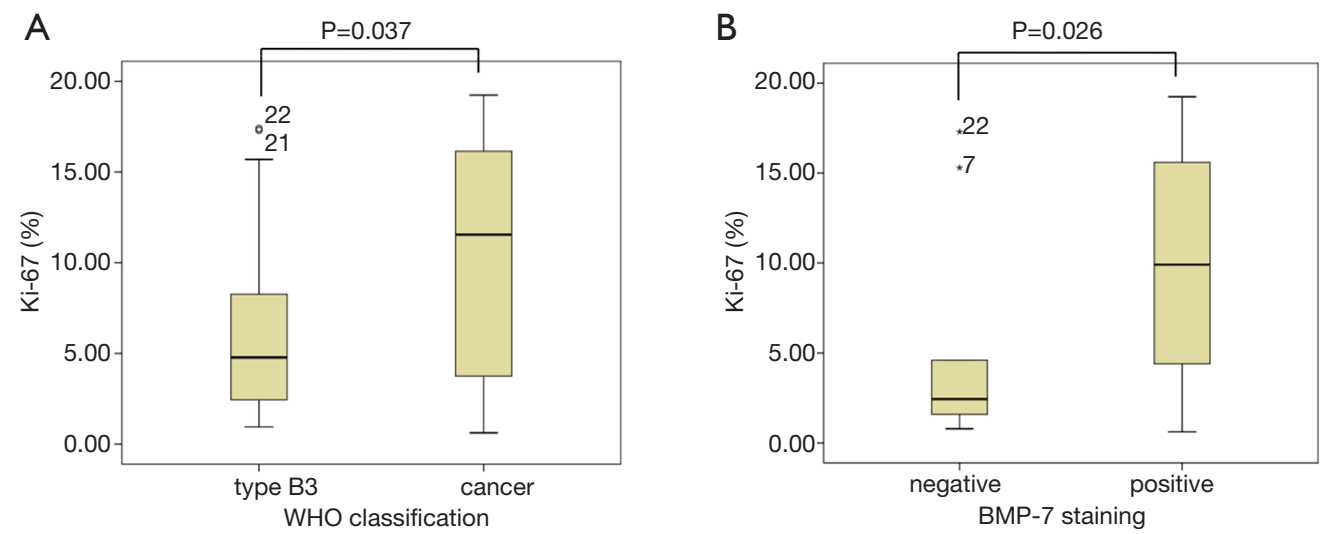

Figure 3 Comparison of Ki-67. (A) Positive ratios in thymoma B3 and thymic cancer and (B) bone morphogenetic protein 7 (BMP-7).

A

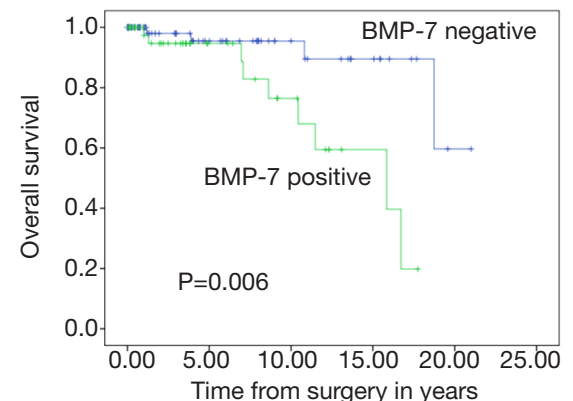

Number at risk

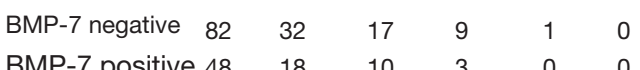

C

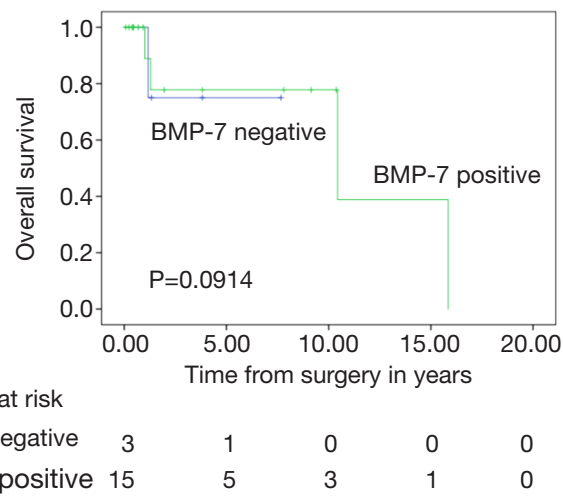

B

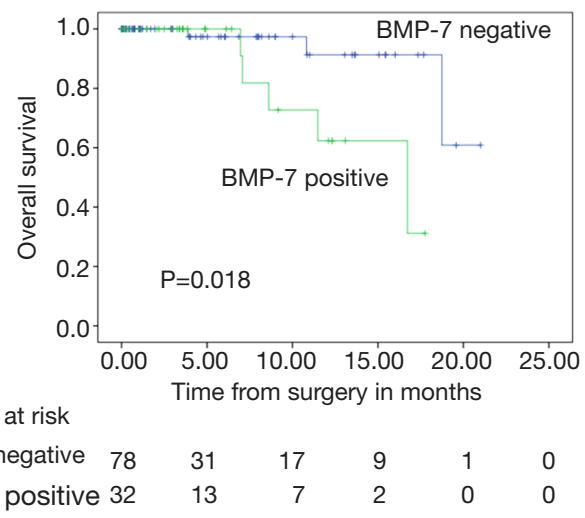

\begin{tabular}{|c|c|c|c|c|}
\hline MP-7 negative 78 & 31 & 17 & 9 & 1 \\
\hline BMP-7 positive 32 & 13 & 7 & 2 & 0 \\
\hline
\end{tabular}

Figure 4 Overall survival curves all patients with thymic epithelial tumors (A), patients with thymoma (B), and thymic cancer (C).

thymoma and thymic cancer, which indicated that thymic epithelial tumors with high BMP-7 expression might have an aggressive phenotype, and that the expression of BMP-7 could reflect high tumor malignancy. Thus, this is the first report that demonstrates the significance of BMP-7 as a biomarker in distinguishing aggressive types of thymic epithelial tumors.

Previous studies indicated that high expression of BMP-7 correlated with a poor prognosis in gastric cancer, colorectal cancer, hepatocellular cancer, malignant melanoma, and esophageal carcinoma (14-18). Recently, the relationship between the expression of BMP-7 and 
Table 3 Univariate and multivariate Cox hazards regression analyses of the clinicopathological parameters in all thymic epithelial tumor patients

\begin{tabular}{|c|c|c|c|c|c|c|}
\hline Variable & \multicolumn{3}{|c|}{ Univariate } & \multicolumn{3}{|c|}{ Multivariate } \\
\hline Age (<65 vs. $\geq 65)$ & 8.784 & 2.339-32.994 & 0.001 & 4.617 & $1.128-18.9$ & 0.033 \\
\hline Sex (male vs. female) & 1.16 & $0.671-2.007$ & 0.595 & & & \\
\hline Tumor size ( $<40$ vs. $\geq 40 \mathrm{~mm})$ & 0.567 & $0.153-2.106$ & 0.396 & & & \\
\hline WHO histological grade (thymic cancer vs. thymoma) & 7.177 & $2.129-24.197$ & 0.001 & 2.149 & $0.48-9.62$ & 0.317 \\
\hline BMP-7 staining (positive vs. negative) & 5.181 & $1.397-19.211$ & 0.014 & 2.115 & $0.503-8.89$ & 0.307 \\
\hline
\end{tabular}

$\mathrm{HR}$, hazard ratio; $\mathrm{Cl}$, confidence interval; BMP-7, bone morphogenetic protein-7.

epithelial mesenchymal transition (EMT) was reported. Lim et al. demonstrated that expression of E-cadherin was reduced by the addition of BMP-7 in prostate cancer cells (19). They showed that BMP-induced activation of PI3K and Erk contributed to EMT morphologic conversion of the prostate cancer cells. Xu et al. demonstrated that inhibition of BMP-7 using siRNA lead to up-regulation of E-cadherin and down-regulation of MMP-9 in esophageal squamous cell lines (18). Thus, the involvement of BMP-7 in the regulation of cell migration and metastasis through modulation of EMT has been reported in various malignant tumors. In the present study, the expression of BMP-7 expression was significantly higher in type $\mathrm{B} 3$ thymoma and thymic cancer than in type $\mathrm{A}, \mathrm{AB}, \mathrm{B} 1$, and $\mathrm{B} 2$ thymomas. Furthermore, patients with BMP-7 positive tumors showed significantly poorer OS than those with BMP-7 negative tumors in all patients with thymic epithelial tumors. These findings indicate that BMP-7 might be a negative prognostic factor in thymic epithelial tumors, as well as in the other malignancies reported previously.

$\mathrm{Ki}-67$ is one of the universal markers of cell proliferation, and the correlation between a high ratio of $\mathrm{Ki}-67$ positive cells in the tumor and the unfavorable prognosis of the patients has been reported in various malignancies (20-25). The relationship between Ki-67 staining and thymic epithelial tumors has been reported in previous studies $(7,26)$; however, the usefulness of Ki-67 staining in the differential diagnosis of thymic epithelial tumors has not been established. Our study demonstrated that there was a significant difference in the ratio of $\mathrm{Ki}-67$ expression between type B3 thymoma and thymic cancer. In addition, tumors with high levels of BMP-7 expression showed significantly higher ratios of Ki-67 positive cells in type B3 thymoma and thymic cancer. Thus, our study indicated that BMP-7 expression could be correlated with cell proliferation and might reflect the aggressive phenotype observed in type B3 thymoma and thymic cancer.

Generally, patients with thymic cancer have a poorer prognosis than those with thymoma; however, it is often difficult to distinguish thymic cancer from type B3 thymoma pathologically. Since the expression of BMP-7 did not differ between thymic cancer and type B3 thymoma in the present study, this protein may not be useful in the differential diagnosis of these thymic epithelial tumors. However, the expression of BMP-7 in type B3 thymoma was significantly higher than in other types of thymomas. Type B1, B2, and B3 thymomas are generally distinguished by the number of background lymphocytes. A major feature of type B3 thymoma is the overwhelming majority of round epithelial tumor cells with conspicuous cytological atypia and fewer lymphocytes. Okumura et al. reported that the prognosis of patients with type B3 thymoma was significantly worse than that of those with type B1 and B2 thymoma (27). In the present study, the ratio of positive expression of BMP-7 in type B3 thymoma was similar to that in thymic cancer. These findings indicate the possibility that BMP-7 might serve as an adjunct marker to distinguish aggressive types of thymoma.

Previous studies indicated that over-expression of BMP-7 was associated with lymph node metastasis and proliferation in melanoma (17) and was correlated with the incidence of liver metastasis in colorectal cancer (15). Thus, the expression of BMP-7 could be associated with progression and metastasis in malignant neoplasms. However, in this study, there was no relationship between the expression of BMP-7 and Masaoka stage, which classifies thymomas based on invasiveness of the tumor through the capsule into the surrounding tissue, organs, and metastases. In contrast, 
a significant correlation between the expression of BMP-7 and the WHO classification, which was based on the histomorphological findings, was observed in the present study. These findings suggest that BMP-7 might contribute to the biological behavior of tumor cells in thymic epithelial tumors.

In the present study, univariate analyses showed that Masaoka stage, WHO classification, and BMP-7 expression were significant predictors of OS in all patients with thymic epithelial tumors. However, none of these factors were significant predictors in multivariate analyses. These findings indicate that each classification alone was not sufficient for the prediction of the biological behavior of thymic epithelial tumors.

There are some limitations in this study. Firstly, there is no available cultured thymoma cell line by which we could analyze the role of BMP-7 in thymic epithelial cells in vitro. Secondly, as the prognosis of the patients with thymic epithelial tumors was relatively favorable and there were a limited number of patients in this study, we could not compare the recurrence rate or tumor specific death rate according to the expression of BMP-7. It is necessary to increase the number of cases and analyze the relationship between BMP-7 and other factors to establish the clinical usefulness of BMP-7 as a diagnostic biomarker in thymic epithelial tumors.

\section{Conclusions}

In conclusion, this study showed that high expression of BMP-7 correlated with the poor prognosis of patients with thymic epithelial tumors, and the expression of BMP-7 was higher in type $\mathrm{B} 3$ thymomas and thymic cancers than in other types of thymomas. Although further experiments, including in vitro analyses, are required to analyze the function of BMP-7 in thymic epithelial tumors, our study indicates that BMP-7 might serve as a novel prognostic biomarker for thymic epithelial tumors, as its expression reflects the high proliferative ability and aggressiveness of thymic epithelial tumors.

\section{Acknowledgments}

Funding: None.

\section{Footnote}

Conflicts of Interest: The authors have no conflicts of interest to declare.

Ethical Statement: The authors are accountable for all aspects of the work in ensuring that questions related to the accuracy or integrity of any part of the work are appropriately investigated and resolved. This study was approved by Shinshu University Research Ethics Committee (No. 542).

Open Access Statement: This is an Open Access article distributed in accordance with the Creative Commons Attribution-NonCommercial-NoDerivs 4.0 International License (CC BY-NC-ND 4.0), which permits the noncommercial replication and distribution of the article with the strict proviso that no changes or edits are made and the original work is properly cited (including links to both the formal publication through the relevant DOI and the license). See: https://creativecommons.org/licenses/by-nc-nd/4.0/.

\section{References}

1. Roden AC. Evolution of Classification of Thymic Epithelial Tumors in the Era of Dr Thomas V. Colby. Arch Pathol Lab Med 2017;141:232-46.

2. Strobel P, Marx A, Zettl A, et al. Thymoma and thymic carcinoma: an update of the WHO Classification 2004. Surg Today 2005;35:805-11.

3. Tateyama H, Sugiura H, Yamatani C, et al. Expression of podoplanin in thymoma: its correlation with tumor invasion, nodal metastasis, and poor clinical outcome. Hum Pathol 2011;42:533-40.

4. Cui F, He J, Liu J, et al. Protein expression status of p53 and epidermal growth factor receptor in thymoma. Oncol Lett 2011;2:459-63.

5. Pan CC, Ho DM, Chen WY, et al. Ki67 labelling index correlates with stage and histology but not significantly with prognosis in thymoma. Histopathology 1998;33:453-8.

6. Kojika M, Ishii G, Yoshida J, et al. Immunohistochemical differential diagnosis between thymic carcinoma and type B3 thymoma: diagnostic utility of hypoxic marker, GLUT-1, in thymic epithelial neoplasms. Mod Pathol 2009;22:1341-50.

7. Yokota K, Tateyama H, Yano M, et al. Clinicopathological analysis of small-sized thymoma with podoplanin and $\mathrm{Ki}$ 67 expression analysis. Mol Clin Oncol 2013;1:88-92.

8. Ozkaynak E, Rueger DC, Drier EA, et al. OP-1 cDNA encodes an osteogenic protein in the TGF-beta family. EMBO J 1990;9:2085-93. 
9. Dudley AT, Lyons KM, Robertson EJ. A requirement for bone morphogenetic protein-7 during development of the mammalian kidney and eye. Genes Dev 1995;9:2795-807.

10. Luo G, Hofmann C, Bronckers AL, et al. BMP-7 is an inducer of nephrogenesis, and is also required for eye development and skeletal patterning. Genes Dev 1995;9:2808-20.

11. Zouvelou V, Luder HU, Mitsiadis TA, et al. Deletion of BMP7 affects the development of bones, teeth, and other ectodermal appendages of the orofacial complex. J Exp Zool B Mol Dev Evol 2009;312B:361-74.

12. Patel SR, Dressler GR. BMP7 signaling in renal development and disease. Trends Mol Med 2005;11:512-8.

13. Milan M. Sculpting a fly leg: BMP boundaries and cell death. Nat Cell Biol 2007;9:17-8.

14. Aoki M, Ishigami S, Uenosono Y, et al. Expression of BMP-7 in human gastric cancer and its clinical significance. Br J Cancer 2011;104:714-8.

15. Motoyama K, Tanaka F, Kosaka Y, et al. Clinical significance of BMP7 in human colorectal cancer. Ann Surg Oncol 2008;15:1530-7.

16. Li W, Cai HX, Ge XM, et al. Prognostic significance of BMP7 as an oncogene in hepatocellular carcinoma. Tumour Biol 2013;34:669-74.

17. Rothhammer T, Wild PJ, Meyer S, et al. Bone morphogenetic protein 7 (BMP7) expression is a potential novel prognostic marker for recurrence in patients with primary melanoma. Cancer Biomark 2007;3:111-7.

18. Xu G, Tang S, Yang J, et al. BMP7 expression in esophageal squamous cell carcinoma and its potential role in modulating metastasis. Dig Dis Sci 2013;58:1871-9.

19. Lim M, Chuong CM, Roy-Burman P. PI3K, Erk signaling in BMP7-induced epithelial-mesenchymal

Cite this article as: Miura $\mathrm{K}$, Hamanaka $\mathrm{K}$, Uehara $\mathrm{T}$, Momose M, Kanai Y, Matsuoka S, Takeda T, Agatsuma H, Hyogotani A, Ito KI. Bone morphogenetic protein-7 expression reflects the high proliferative ability and aggressiveness of thymic epithelial tumors. J Thorac Dis 2020;12(3):448-456. doi: $10.21037 /$ jtd.2020.01.14 transition (EMT) of PC-3 prostate cancer cells in 2- and 3-dimensional cultures. Horm Cancer 2011;2:298-309.

20. Melling N, Kowitz CM, Simon R, et al. High Ki67 expression is an independent good prognostic marker in colorectal cancer. J Clin Pathol 2016;69:209-14.

21. He X, Chen Z, Fu T, et al. Ki-67 is a valuable prognostic predictor of lymphoma but its utility varies in lymphoma subtypes: evidence from a systematic meta-analysis. BMC Cancer 2014;14:153.

22. Tian Y, Ma Z, Chen Z, et al. Clinicopathological and Prognostic Value of Ki-67 Expression in Bladder Cancer: A Systematic Review and Meta-Analysis. PLoS One 2016;11:e0158891.

23. Wen S, Zhou W, Li CM, et al. Ki-67 as a prognostic marker in early-stage non-small cell lung cancer in Asian patients: a meta-analysis of published studies involving 32 studies. BMC Cancer 2015;15:520.

24. Sofocleous CT, Garg SK, Cohen P, et al. Ki 67 is an independent predictive biomarker of cancer specific and local recurrence-free survival after lung tumor ablation. Ann Surg Oncol 2013;20 Suppl 3:S676-83.

25. Sofocleous CT, Garg S, Petrovic LM, et al. Ki-67 is a prognostic biomarker of survival after radiofrequency ablation of liver malignancies. Ann Surg Oncol 2012;19:4262-9.

26. Yang WI, Efird JT, Quintanilla-Martinez L, et al. Cell kinetic study of thymic epithelial tumors using PCNA (PC10) and Ki-67 (MIB-1) antibodies. Hum Pathol 1996;27:70-6.

27. Okumura M, Ohta M, Tateyama H, et al. The World Health Organization histologic classification system reflects the oncologic behavior of thymoma: a clinical study of 273 patients. Cancer 2002;94:624-32. 